\title{
Multi-agent Architecture for Reconfiguration of Precision Modular Assembly Systems
}

\author{
Pedro Ferreira, Niels Lohse, and Svetan Ratchev \\ The University of Nottingham, Precision Manufacturing Centre, Nottingham, \\ NG7 2RD, United Kingdom \\ Tel.: +44 1159513875 \\ epxpf@nottingham.ac.uk
}

\begin{abstract}
Precision assembly systems today are subject to high levels of change which require fast physical and logical adaption. As such, new methods and tools need to reflect this need providing adaptive systems that can react to market changes in a timely and cost effective manner. Towards achieving increased responsiveness several developments of new modular concepts provided the bases for higher system adaptability through increased module interchangeability and reusability. The modularization of physical and control infrastructure only solves one aspect of the issue, there is still a lack of appropriate tools and methods to support requirements driven reconfiguration of such systems. This paper proposes an agent architecture for reconfiguration of equipment modules driven by a set of requirements. A new agent model is proposed which addresses the specific needs of precision modular assembly systems catering both for physical and logical constraints of the modules as well as their joint emergent behaviour.
\end{abstract}

Keywords: Reconfigurable Assembly Systems, Modular Assembly Systems.

\section{Introduction}

The question of component reusability and dynamic reconfiguration of precision assembly systems has become increasingly more important due to ever decreasing product life-cycles, increasing product variants and rising process complexity. General purpose assembly machines, equivalent to $\mathrm{CNC}$ machine tools, are only available in specialist domains such as printed circuit board assembly where the components are highly standardised. The assembly of most other products demand custom made systems which address the specific requirements for these products. Today, these are mostly "Engineered to Order" making them cost and time intensive to design or reengineer. Reconfiguration of assembly systems requires a certain level of flexibility. This can be achieved by adding more equipment to the system, however adding equipment for future use can be very costly. Modular systems offer the structure to add or remove equipment as and when it is needed, thus enabling system capabilities to be continuously adapted to changing requirements over the lifecycle of the system. Increased modularisation of assembly equipment, rapid integration mechanisms, and 
automatic configuration tools are considered fundamental for the move towards cost and time effective configuration and re-configuration of complex assembly systems $[1,2,3]$.

Significant effort has been directed towards creating modular assembly system (MAS) architectures for physical equipment and control interchange ability. The EU project EUPASS, for instance, has created a framework for rapid integration for ultraprecision assembly modules defining hardware interfaces, control interfaces, and module description formats [4]. Other modular assembly system platforms have been proposed $[5,6]$. While the number and completeness of underlying industrial applicable standards is still limited, there is a clear drive to overcome this barrier.

Standardisation of hardware and software interfaces is, however, only one aspect of rapid assembly system reconfiguration. Effective tools and methods for the requirements driven system analysis, reconfiguration and validation of complex assembly system solutions are also needed to drastically reduce the time and effort required for the development of highly dedicated assembly systems. MAS reconfiguration methods reported today adopt a human driven approach, which is based on the expertise of the user. These methods provide valid solutions, however, very few of these are replicable and transparent, and are constrained by the individual knowledge of the user.

This paper proposes a multi-agent architecture for reconfiguration of precision MAS based on the changed product and process requirements. The proposed architecture is directed towards utilising latest agent-based negotiation protocols to enable the scalable and extendable requirements driven reconfiguration of complex MAS assessing the reconfiguration effort and providing solutions from the existing modules if possible. The system can also access a library of existing modular building blocks to enhance the system with the required functionality.

\section{Literature Review}

The term reconfiguration implies that something has been configured and requires a new configuration. The basic principles of a configuration still apply a reconfiguration, although the decision making process will differ quite substantially. The use of different methodologies for configuration design depends on the complexity of the system [7], as for the reconfiguration. Systems can be classified as an uncoupled system, loosely-coupled system, or strongly-coupled system. MAS are normally comprised of strongly-coupled and loosely-coupled systems. A reconfiguration methodology for MAS has to cater solutions for the presence of both.

Reconfiguration can be seen as an enhanced configuration problem, e.g. the addiction of extra requirements and constraints. Configuration design of MAS is comparable to the design of modular products [7]. Therefore there are many methods that can be applied such as feature-based methods [8], hierarchical decomposition methods [9], combinatorial synthesis method [10], entity-based methods [11], and case-based methods [12]. In strongly-coupled systems all design variables have to be considered together to validating if the configuration fulfils its requirements [7].

The configuration design at system level is usually achieved through a propose-andtest approach in conjunction with system simulation software for testing. An approximate solution is found in a time-consuming iterative process. Mathematical formulation 
for the system level would be too complex and it is usually only used for specific subproblems [7]. Deterministic models where the system variables are constant have been used in configuration design [13], these do, however, limit the flexibility of the design system. Stochastic models arise as a solution to this problem since they provide at least one uncertain variable. Some configuration design methodologies have used stochastic models in order to deal with the configuration problems [14, 15].

The research on reconfiguration of assembly systems is mostly in the control aspects of the assembly systems producing reconfigurable software which is able to change the control of the assembly systems yet falling short on physical reconfiguration and system enhancement. These approaches are also not related with the systems design and requirements specifications, and are mostly human driven.

Despite the significant work in the area of configuration and reconfiguration methods, there is still a lack a systematic reconfiguration methodology that caters for different reconfigurable machines and is driven by design requirements [16]. Furthermore, most of the proposed methods in the field of manufacturing system reconfiguration have been focused on the machine level, while the systems have been designed largely intuitively [7].

Agent technology is seen as the natural way to address the problems of scalability and flexibility manufacturing systems. As a result agent technology has been widely applied to provide solutions in the manufacturing domain [17]. This provides extensive literature in agent models, negotiation models, agent environments, etc, however these models are mostly application specific.

The exiting agent-based approaches are mainly focussed on providing agility and low level reconfigurability. For reconfiguration design, they also need to have the tendency to provide close to optimal solutions. The optimization in distributed systems is quite different from other approaches which target global optimization through mathematical formulation of the whole problems. The mathematical formulation of complex system is quite difficult to develop and maintain, thus are mainly successful for simpler systems. Agent approaches on the other hand attempt to achieve optimization through efficient coordination mechanisms and thus require significantly simpler models [17]. Despite all the work done in this field there is still no agent architecture which focuses on the specific issues of modular assembly systems identified by [18], namely providing clear formalisms for equipment capability and interconnection constraint representation as well as methods and protocols for system formation from these modules based on a given set of requirements.

\section{Problem Definition}

The redesign of assembly systems today is a largely human driven process relaying heavily on the skill and expertise of system integrators. This process can provide valid system reconfigurations, however, it seldom follows a systematic approach and as a result will often be quite expensive and time consuming, making frequent system adaptations infeasible. Furthermore, there is a lack of clear assessment methods and tools which can establish when a reconfiguration should occur.

The MAS paradigm with its focus on clear functional decoupling of equipment module functionalities and standardised interfaces for interchange ability has opened 
the scope for automatic reconfiguration methods. Thus, it is possible to clearly formalise the functional capabilities and connectivity constraints of the available modules hence allowing the mapping of required against available capabilities. The redesign of MAS is essentially a restructure of equipment and assembly process, across several levels of granularity with equipment modules and their functional capabilities as the elementary building blocks.

The MAS reconfiguration problem is centre on two questions "when to reconfigure?" and "how to reconfigure?". Current solutions rely on the system integrator to assess the change in the system requirements and cater for the new requirements, reconfiguring the system accordingly while making decision about the added benefit resulting from reconfigurations.

A reconfiguration methodology for MAS has to be able to assess and recommend whether an existing system should be maintained in its current configuration or should be adapted in some form to provide the best cost benefit for a new set of requirements. Within a MAS paradigm, the reconfiguration methodology should start with the existing system and the changes in the requirements, and base all its recommendations on the available set of equipment modules as illustrated in Fig. 1.

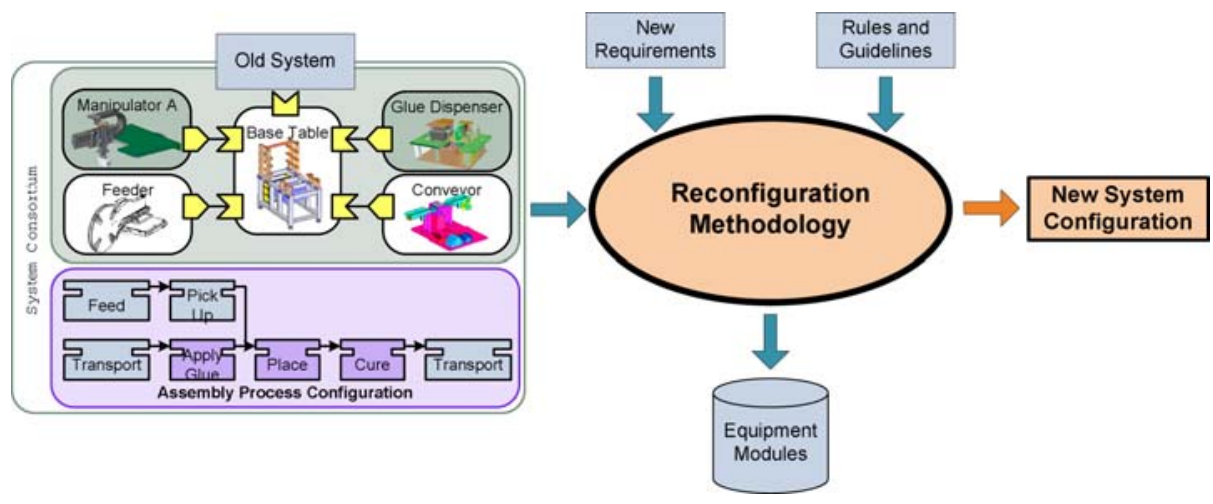

Fig. 1. Problem definition overview

\section{Agent Architecture}

This section gives a detailed overview of the proposed agent-architecture for MAS reconfiguration and explains the rational behind it. The GAIA methodology developed by [19] has been applied to translate the requirements identified for MAS reconfiguration problems above into an appropriate agent architecture. A schematic overview of the resulting agent architecture is given in Fig. 2.

The clear common denominator of all reconfiguration design methodologies including for MAS is the need to elicit and maintain the system requirements independent of the proposed solution alternatives. Consequently there is a need for Requirements Agents which are able to provide clear objectives to those agents involved in the reconfiguration process. Furthermore, they need to be able to represent the interests of the customer/system user to validate possible system reconfigurations. 


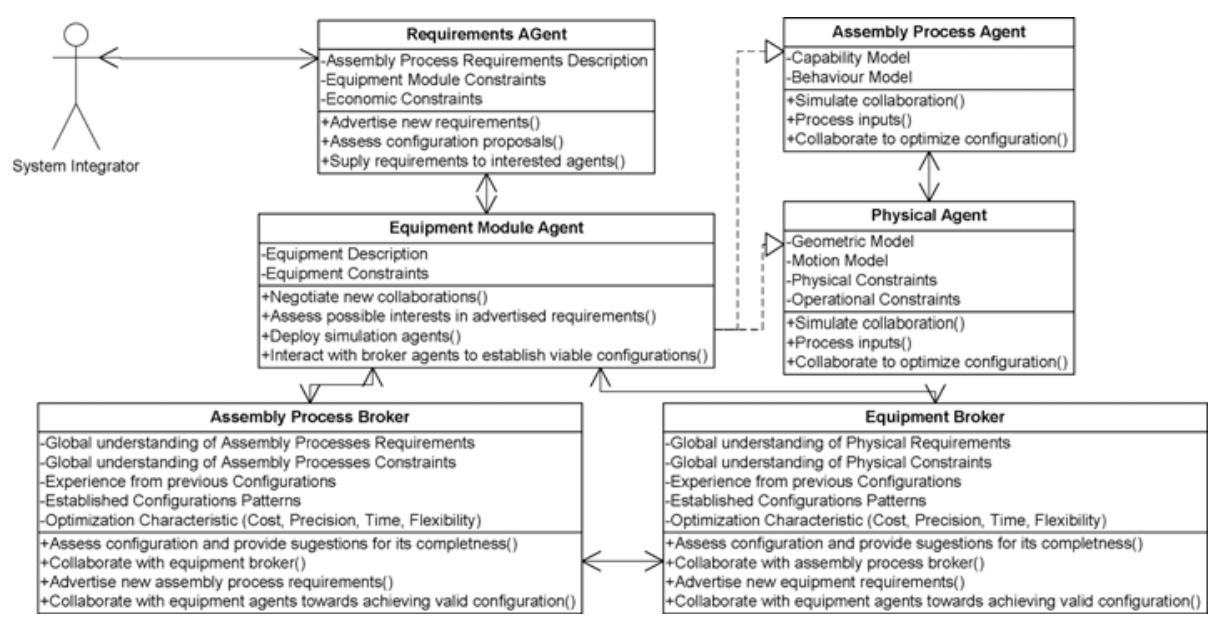

Fig. 2. Agent architecture for reconfigurable assembly systems

Another important set of actors within this problem domain are the equipment modules. It is proposed that each equipment module should be represented by an Equipment Module Agent that has a detailed understanding of the module's capabilities and behaviour. The role of mediator has been assigned to two types of agents as the configuration of MAS always needs to address both the equipment and the process: the Physical Broker Agent, and the Assembly Process Broker agents. Both are specialised to validate the logical constraints of physical and assembly process configurations respectively. These agents will negotiate tradeoffs between themselves in order to balance the equipment reconfiguration and assembly process reconfiguration constraints. This architecture requires some early evaluation of the likely success of reconfiguration within the existing consortium. Thus some method for early evaluation of the likely success the reconfiguration within the consortium needs to be available to reduce the computation effort. To provide some bases for early comparison, it is proposed that the Equipment Module Agents deploys Physical and Assembly Process Agents into a simulation environment. They represent the physical and process capabilities of the modules and dynamically interact with each other to determine the emergent behaviour and performance characteristics of a consortium. This will also provide valuable input for the benefits vs. effort analysis to assess if the reconfiguration should occur.

The whole process of reconfiguration is triggered by the requirements agents. This agent approaches the Equipment Module Agents involved in the established consortium with new requirements. The next step in the reconfiguration process happens through the negotiation of the Equipment Module Agents involved in the consortium and the Broker Agents that provide the expert knowledge for the reconfiguration. If the consortium is unable to provide a new solution, the broker agents will provide expert knowledge to enable phase two, the contact of other Equipment Module Agents that can enhance the consortium towards fulfilling the new requirements. This is followed by the negotiation between new and old equipment module agents and brokers to establish possible system alternatives. Once these system alternatives have 
been identified the Physical and Assembly Process Agents are deployed to provide the assessment of the different alternatives and determine their validity.

\section{Illustrative Example}

This section shows the application of the proposed agent architecture with the help of an illustrative example, which is both simple enough to follow and complex enough to show the potential of the architecture. Let us assume an automatic workstation for the gluing of two components requires a reconfiguration for a new product (Fig. 1).

The user or system integrator would start a new Requirements Agent and specify the new desired system characteristics. In this case it would simply be a new set of characteristics for the joining of the two components. For simplicity it is assumed that only the precision requirement is changed.

The first step for the system in the reconfiguration effort is to assess if it is possible to reconfigure using the same modules, since this would require the least amount of change. In this situation this analysis determines that all the modules have the minimum precision required. However once the Equipment Module Agents deploy the Assembly Process Agents for the assembly process simulation, it is determined that the errors stack up above the requirements for precision.

The Broker Agent is then contacted to provide strategies to compensate for lack of precision, namely the increase of precision by replacing less precise modules, or add measuring capabilities to compensate for stacked up errors. Given that the Assembly Process Agent during their analysis of the existing system have already determined which module is the bottleneck, the Equipment Module Agents can then proceed to finding alternatives based on the Broker Agent suggested alternatives. For the given example the bottleneck assembly process is "Place" which is performed by "Manipulator A". As such, the consortium will either find a way to compensate for the lack of precision by enhancing the system with a measuring module, or by replacing this module.

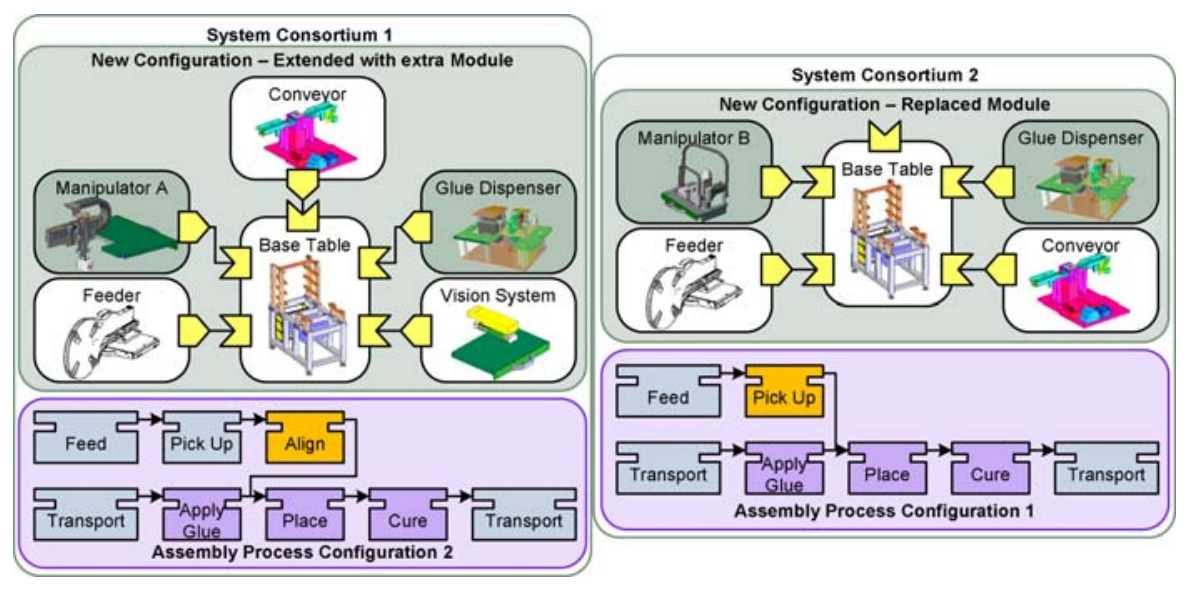

Fig. 3. Example of Alternative MAS Configurations 
The Equipment Module Agents based on this knowledge advertise new module requirements for the system, which are in turn answered by relevant Equipment Module Agents outside of the original consortium. The possible alternatives are then evaluated based on their value to the consortium, and the most promising ones are simulated through the deployment of the Assembly Process Agents and Physical Agents. In this example the system would produce two promising reconfigurations for the system; adding a vision system to compensate for error; or replacing the manipulator with a more accurate one (Fig. 3). Because in both situations the system requires both physical and logical changes, the effort value is based on cost. By considering the cost of new modules as similar, it is clear that solution 2 has the advantage since the old module could be resold or reused in another system.

\section{Conclusion}

The presented agent architecture demonstrates clear potential to deal with MAS reconfiguration. This is expected to reduce reconfiguration times by better supporting the reconfiguration of MAS. This architecture also supports the constant analysis of a MAS to determine the reconfiguration effort at any given time. It is foreseen that the reconfiguration effort can be used against predicted benefits to determine the best time for reconfiguration.

Further work will focus on the development of agent negotiation and collaboration protocols which address the specific needs of MAS.

\section{Acknowledgement}

The reported work is partially funded by the European Union as part of the NMP-2CT-2004-507978 EUPAS project. The support is gratefully acknowledged.

\section{References}

1. Koren, Y., et al.: Reconfigurable Manufacturing Systems. CIRP Annals - Manufacturing Technology 48(2) (1999)

2. Kratochvíl, M., Carson, C.: Growing Modular. Spinger, Berlin (2005)

3. Onori, M., et al.: European precision assembly roadmap 2012. The Assembly-Net Consotium (2002)

4. EUPASS. Evolvable Ultra-Precsion Assembly SystemS (2008), http: / /www . eupass-fp6.org/

5. Alsterman, H., Onori, M.: Process-Oriented Assembly System Concepts - The MarklV Approach. In: ISATP 2001, Fukuoka, Japan (2001)

6. Gaugel, T., Bengel, M., Malthan, D.: Building a mini-assembly system from a technology construction kit. Assembly Automation 24(1), 43-48 (2004)

7. Bi, Z.M., et al.: Reconfigurable manufacturing systems: the state of the art. International Journal of Production Research 46, 967-992 (2008) 
8. Perremans, P.: Feature-based description of modular fixturing elements: the key to an expert system for the automatic design of the physical fixture. Advances in Engineering Software 25(1), 19-27 (1996)

9. Tsai, Y.-T., Wang, K.-S.: The development of modular-based design in considering technology complexity. European Journal of Operational Research 119, 692-703 (1999)

10. Levin, M.S.: Towards combinatorial analysis, adaptation, and planning of humancomputer systems. Applied Intelligence 16(3), 235-247 (2002)

11. Hong, N.K., Hong, S.: Entity-based models for computer-aided design systems. Journal of Computing in Civil Engineering 12(1), 30-41 (1998)

12. Watson, I.: Case-based reasoning is a methodology not a technology. Knowledge-Based Systems 12, 303-308 (1999)

13. Tang, L., et al.: Concurrent Line-Balancing, Equipment Selection and Throughput Analysis for Multi-Part Optimal Line Design. The International Journal for Manufacturing Science \& Production 6, 71-81 (2004)

14. Ohiro, T., et al.: A stochastic model for deciding an optimal production order and its corresponding configuration in a reconfigurable manufacturing system with multiple product groups. International Conference on Agile, Reconfigurable Manufacturing (2003); Ann Arbor

15. Zhao, X., Wang, K., Luo, Z.: A stochastic model of a reconfigurable manufacturing system Part I, a framework. International Journal of Production Research 38(10), 2273-2285 (2000)

16. Bi, Z.M., et al.: Development of reconfigurable machines. The International Journal of Advanced Manufacturing Technology (2007)

17. Shen, W., et al.: Applications of agent-based systems in intelligent manufacturing: An updated review. Advanced Engineering Informatics 20(4), 415-431 (2006)

18. Lohse, N.: Towards an Ontology Framework for the Integrated Design of Modular Assembly Systems. University of Nottingham (2006)

19. Zambonelli, F., Jennings, N.R., Wooldridge, M.: Multiagent systems as computational organisations: the Gaia methodology. In: Henderson-Sellers, B., Giorgini, P. (eds.), pp. 136171. Idea Group Publishers (2005) 